\title{
Arctiinae (Lepidoptera, Arctiidae) coletados em unidades de conservação estaduais do Rio Grande do Sul, Brasil
}

\author{
José Augusto Teston ${ }^{1}$, Alexandre Specht ${ }^{2}$, Rocco Alfredo Di Mare ${ }^{3} \&$ Elio Corseuil $^{4}$
}

\begin{abstract}
${ }^{1}$ Campus de Altamira, Colegiado de Biologia-UFPA, Rua Cel. José Porfírio s/n, 68370-000 Altamira-PA, Brasil - jateston@gmail.com ${ }^{2}$ Departamento de Ciências Exatas e da Natureza, UCS-Curso de Ciências-Biologia, Alameda João Dal Sasso 800, 95700-000 Bento GonçalvesRS, Brasil; Programa de Pós-Graduação em Biotecnologia, Instituto de Biociências, Rua Francisco Getúlio Vargas 1130, $95070-560$ Caxias do Sul - Brasil. aspecht@ucs.br

${ }^{3}$ Departamento de Biologia-UFSM, CCNE, Campus Universitário, km 9 Faixa de Camobi, 97119-900 Santa Maria-RS, Brasil. ram13@terra.com.br ${ }^{4}$ Faculdade de Biociências-PUCRS, Caixa Postal 1429, 90619-900 Porto Alegre-RS, Brasil. corseuil@pucrs.br
\end{abstract}

\begin{abstract}
Arctiinae (Lepidoptera, Arctiidae) collected in protected unities in Rio Grande do Sul State, Brazil. The objective of this work was to analyze the composition of the Arctiinae communities at Parque Estadual do Espigão Alto (Barracão), Parque Estadual de Rondinha (Sarandí), Parque Estadual do Turvo (Derrubadas) and at Reserva Biológica de Ibirapuitã (Alegrete). Ten light traps were used to collect the lepidopterous from November/2000 to February/2001, sampling one nigth per area. The lepidopterous were identified at subfamily and tribe levels and, when it was possible, at genera and species levels. A total 5,969 Arctiinae belonging to 137 morpho species, distributed in 6 tribes were collected.
\end{abstract}

KEYWORDS. Biodiversity; community structure; faunistic inventory; light-trap.

RESUMO. Arctiinae (Lepidoptera, Arctiidae) coletados em unidades de conservação estaduais do Rio Grande do Sul, Brasil. O presente estudo foi realizado com o objetivo de analisar a composição das comunidades de Arctiinae no Parque Estadual do Espigão Alto (Barracão), Parque Estadual de Rondinha (Sarandí), Parque Estadual do Turvo (Derrubadas) e na Reserva Biológica de Ibirapuitã (Alegrete). Dez armadilhas luminosas foram utilizadas para coletar os lepidópteros, de novembro de 2000 a fevereiro de 2001, amostrando-se uma noite em cada local. Os lepidópteros foram identificados aos níveis de subfamília, tribo e, quando possível gênero e espécie. Obteve-se um total de 5.969 arctiíneos pertencentes a 137 morfo espécies, distribuidos em seis tribos.

PALAVRAS-CHAVE. Armadilha luminosa; biodiversidade; estrutura da comunidade; inventariamento de fauna.

Os lepidópteros estão entre as principais ordens de insetos destacando-se quanto à riqueza de espécies, importância econômica e distribuição em quase todos os ambientes do planeta. O grupo tem sido utilizado em estudos ambientais através da mensuração da biodiversidade (Intachat \& Woiwod 1999; Landau et al. 1999; Kitching et al. 2000; Hammond \& Miller 1998), também podem servir como indicadores da vegetação em diferentes tipos de hábitats, pois em sua grande maioria são fitófagos (Kitching et al. 2000).

A utilização de armadilhas luminosas para captura de mariposas é muito difundida entre os pesquisadores, existindo estudos sobre modelos e fontes luminosas (Muihead-Thompson 1991; Fry \& Waring 1996). Face à influência dos fatores ambientais e do comprimento de onda da luz emitida pelas lâmpadas sobre a atração das mariposas (Marinoni \& Dutra 1996; Butler et al. 1999) há necessidade de precisar tais aspectos em cada caso para permitir as comparações dos resultados. Vários estudos sobre lepidópteros noturnos utilizando este método de captura, incluindo Arctiidae, já foram realizados no Brasil (Dorval et al. 1995; Ferreira et al. 1995; Lübeck et al. 1995; Pereira et al. 1995; Marinoni \& Dutra 1996; Marinoni et al. 1997; Camargo 1999; Bittencourt et al. 2003 e Teston \& Corseuil 2004).

Apesar de existirem algumas espécies de hábito diurno, os arctiídeos estão entre os lepidópteros noturnos mais utilizados como bioindicadores no monitoramento de ecossistemas, juntamente com Saturniidae e Sphingidae (Hilty \& Merenlender 2000). Este grupo já foi utilizado em estudos anteriores por Teston \& Corseuil (2004) para análise da composição e diversidade em seis zonas fisiográficas do Rio Grande do Sul.

O presente trabalho dá continuidade ao levantamento sobre os lepidópteros noturnos, iniciado com noctuídeos (Specht et al. 2005), analisando agora representantes de Arctiinae ocorrentes em quatro Unidades de Conservação no Estado do Rio Grande do Sul.

\section{MATERIALE MÉTODOS}

As coletas foram realizadas nas Unidades de Conservação Parque Estadual de Espigão Alto (PEE), Parque Estadual de Rondinha (PER), Parque Estadual do Turvo (PET) e Reserva Biológica do Ibirapuitã (RBI), localizadas nos municípios de Barracão (entre as coordenadas $27^{\circ} 30^{\prime}$ e $27^{\circ} 45^{\prime}$ S S 51 $51^{\circ} 20^{\prime}$ e $51^{\circ} 40^{\prime}$ W, com área de $1.331,9$ ha.), Sarandí ( $27^{\circ} 45^{\prime}$ e $27^{\circ} 58^{\prime} \mathrm{S}$; $52^{\circ} 45^{\prime}$ e $52^{\circ} 55^{\prime} \mathrm{W}$, com área de 1.000 ha.), Derrubadas ( $27^{\circ} 00^{\prime}$ e $27^{\circ} 20^{\prime} \mathrm{S}$; $53^{\circ} 40^{\prime}$ e $54^{\circ} 10^{\prime} \mathrm{W}$, com área de $17.491,40$ ha.) e Alegrete (295 $54^{\prime}$ e $29^{\circ} 57^{\prime} \mathrm{S} ; 5^{\circ} 45^{\prime}$ e $55^{\circ} 48^{\prime} \mathrm{W}$, com área de 351,42 ha.), respectivamente, mantidas pelo Departamento Estadual de Florestas e Áreas Protegidas (DEFAP) da Secretaria 
Estadual do Meio Ambiente (SEMA) do Rio Grande do Sul. A caracterização de cada Unidade de Conservação encontrase descrita em Specht et al. (2005). As coletas foram efetuadas empregando armadilhas luminosas, modelo Pensilvânia (Frost 1957), distribuídas em pontos representativos dos vários tipos de vegetação ocorrentes em cada Unidade de Conservação, foram instaladas a cerca de 2,0 m do nível do solo e ficaram ligadas do ocaso até a manhã seguinte, aproximadamente 12 horas. As coletas foram realizadas nas noites de 23/24 de novembro de 2000, na Reserva Biológica do Ibirapuitã; 24/25 de janeiro de 2001, no Parque Estadual do Turvo; 26/27 de janeiro de 2001, no Parque Estadual de Rondinha e, 21/22 de fevereiro de 2001, no Parque Estadual do Espigão Alto. O esforço de coleta compreendeu a utilização de dez armadilhas durante apenas uma noite em cada local.

Os Arctiinae foram separados, quantificados e registrados em planilhas específicas para cada local no Laboratório de Entomologia da PUCRS. Exemplares representativos de cada espécie foram preparados seguindo a metodologia tradicional para Lepidoptera e posteriormente incorporados na coleção entomológica do Museu de Ciências e Tecnologia da Pontifícia Universidade Católica do Rio Grande do Sul (MCTP).

A identificação das espécies foi realizada através de obras de referência (Hampson 1898; 1901; 1914; 1920; Draudt 1915-1917; Seitz 1915-1925; Hering 1925; Watson 1971; $1973 ; 1975 ; 1980)$ e através da comparação com exemplares das coleções do Museu de Ciências da Universidade Católica de Pelotas (MUCP); Museu Entomológico Ceslau Biezanko da Faculdade de Agronomia "Eliseu Maciel", Universidade Federal de Pelotas (MECB); Museu Anchieta de Porto Alegre (MAPA); Museu de Ciências Naturais da Fundação Zoobotânica do Rio Grande do Sul (MCNZ); Museu de Ciências e Tecnologia da PUCRS (MCTP); Museu Ramiro Gomes Costa da Fundação Estadual de Pesquisa Agropecuária (MRGC); Setor de Entomologia da Faculdade de Agronomia da Universidade Federal do Rio Grande do Sul (FASE), de Porto Alegre, RS; Museu Entomológico Fritz Plaumann (MEFP) em Seara, SC; Departamento de Zoologia da Universidade Federal do Paraná(DZUP), em Curitiba, PR; Museu de Zoologia da Universidade de São Paulo (MZSP), em São Paulo, SP; Fundação Instituto Oswaldo Cruz (FIOC); Museu Nacional da Universidade Federal do Rio de Janeiro (MNRJ), no Rio de Janeiro, RJ e também com auxílio de especialistas. Quando necessária a identificação, foi efetuada com a extração e preparo da genitália de espécimes machos.

O posicionamento sistemático adotado segue Kitching \& Rawlins (1999) e Jacobson \& Weller (2002). A distribuição das espécies nos gêneros segue Watson \& Goodger (1986) para as tribos Arctiini, Callimorphini, Phaegopterini e Pericopini, e Hampson (1898), Watson et al. (1995) e Weller et al. (2000) para Ctenuchini e Euchromiini.

Para efeitos de comparação, foi organizada uma listagem apresentando-se a ocorrência e abundância dos arctiíneos, em ordem alfabética de tribos, gêneros e espécies.

\section{RESULTADOS E DISCUSSÃO}

Foram coletados 5.969 exemplares de arctiíneos, pertencentes às tribos com ocorrência registrada para a região Neotropical, Arctiini, Callimorphini, Ctenuchini, Euchromiini, Pericopini e Phaegopterini (Kitching \& Rawlins 1999; Jacobson \& Weller 2002), identificando-se 137 táxons, sendo 106 ao nível específico, 24 ao nível genérico e 7 permanecem apenas ao nível de tribo (Tabela I).

As 106 espécies, perfazem 32,6\% das espécies já registradas para o Rio Grande do Sul (Teston \& Corseuil 2002, 2003a, 2003b, 2004), demonstrando, de forma semelhante ao que já foi observado para Noctuidae (Specht et al. 2005), a importância destas Unidades de Conservação para a preservação dos lepidópteros.

Os arctíneos com maior abundância, acima de 100 espécimes, foram: Paracles variegata (Schaus, 1896), Eucereon sp.2, Bertholdia sp.1 e Symphlebia catenata (Schaus, 1905) no Parque Estadual do Espigão Alto - PEE; Aclytia terra Schaus, 1896 no Parque Estadual do Rondinha - PER; Aclytia heber (Cramer, 1780), Aclytia terra Schaus, 1896, Dysschema amphissa (Geyer, 1832), Dysschema hypoxantha Hübner, 1818, Dysschema picta (Guérin-Méneville, [1844]), Euchlaenidia transcisa (Walker, 1854), Bertholdia sp.1, Leucanopsis umbrosa (Hampson, 1901), Leucanopsis sp.1, Leucanopsis sp.3, Neritos repanda Walker, 1855, Ormetica chrysomelas (Walker, 1856) e Symphlebia catenata (Schaus, 1905) no Parque Estadual do Turvo - PET; e Philoros affinis (Rothschild, 1912) na Reserva Biológica do Ibirapuitã - RBI (Tabela I). No PET houve o maior número de táxons capturados (13) com mais de 100 espécimes, destes também foram capturadas $A$. terra Schaus, no PER e, Bertholdia sp.1 e $S$. catenata (Schaus) ambas no PEE. Todas estas Áreas de Conservação estão localizadas em ambientes de Mata Atlântica (Brasil, 2000).

Ao analisar o material coletado em cada Unidade de Conservação (Tabela II), observou-se que, com um esforço de coleta relativamente pequeno, capturou-se $20,9 \%$ do total das espécies registradas para o Estado no PEE; 20,3\% no PET; 15,4\% no PER e $6,8 \%$ na RBI. Os valores mais elevados ocorreram nas três primeiras Unidades que fazem parte do Bioma Mata Atlântica (Brasil 2000), caracterizado por exuberantes florestas (PEE, PER, PET); já, na RBI, que faz parte do Bioma Campos Sulinos (Brasil 2000), composto por gramíneas, foi encontrado o menor valor. Estes resultados são muito semelhantes aos obtidos, nas mesmas condições com Noctuidae (Specht et al. 2005).

Através da Tabela III observa-se que 43,1\% $(n=59)$ das espécies foram exclusivas. Destas, 51 foram coletadas entre as três unidades presentes no Bioma Mata Atlântica, especialmente $\operatorname{PET}(\mathrm{n}=24)$ e PEE ( $\mathrm{n}=23)$. O menor número de espécies (táxons) exclusivas encontradas em PER $(n=4)$ é atribuído ao seu pequeno tamanho e a forte pressão de uso agrícola em seu entorno como observado com os representantes de Noctuidae (Specht et al. 2005), por outro lado, observou-se que das áreas pertencentes à Mata Atlântica, foi a que apresentou a menor proporção de indivíduos por espécie 11,37. Estes valores, ao contrário do que se observa com Noctuidae (Specht et al. 2005), certamente 
Tabela I. Número de exemplares de Arctiinae capturados com armadilha luminosa em quatro Unidades de Conservação Estaduais do Rio Grande do Sul (PEE - Parque Estadual do Espigão Alto, PER - Parque Estadual de Rondinha, PET - Parque Estadual do Turvo e RBI - Reserva Biológica do Ibirapuitã).

\begin{tabular}{|c|c|c|c|c|}
\hline Tribo e espécies / Unidade de Conservação & PEE & PER & PET & $\mathrm{RBI}$ \\
\hline \multicolumn{5}{|l|}{ Arctiini } \\
\hline 1. Hypercompe cunigunda (Stoll, 1781) & & & 1 & \\
\hline 2. Isia intricata Walker, 1856 & 34 & 32 & 17 & \\
\hline 3. Paracles fusca (Walker, 1856) & 24 & 2 & 5 & 1 \\
\hline 4. Paracles paula (Schaus, 1896) & 5 & 1 & & \\
\hline 5. Paracles variegata (Schaus, 1896) & 158 & 14 & 1 & \\
\hline 6. Paracles sp.1 & 19 & 2 & & 1 \\
\hline \multicolumn{5}{|l|}{ Callimorphini } \\
\hline 8. Utethesia ornatrix (Linnaeus, 1758) & & & & 2 \\
\hline \multicolumn{5}{|l|}{ Ctenuchini } \\
\hline 9. Aclytia heber (Cramer, 1780) & 16 & 34 & 119 & 8 \\
\hline 10. Aclytia jonesi Rothschild, 1912 & 23 & 5 & 37 & \\
\hline 11. Aclytia terra Schaus, 1896 & 26 & 111 & 165 & 12 \\
\hline 12. Argyroeides braco (Herrich-Schäffer, 1855) & & & 1 & \\
\hline 13. Argyroeides flavipes Hampson, 1898 & 2 & & & \\
\hline 14. Argyroeides sanguinea Schaus, 1896 & & 1 & 51 & \\
\hline 16. Cyanopepla jucunda (Walker, 1854) & 5 & & & \\
\hline 17. Delphyre pyroperas Hampson, 1911 & & 5 & 17 & \\
\hline 18. Diptilon doeri (Schaus, 1892) & 5 & & & \\
\hline 19. Diptilon halterata (Fabricius, 1775) & & 1 & 40 & \\
\hline 20. Episcepsis endodasia Hampson, 1898 & 20 & 14 & 15 & \\
\hline 21. Eucereon capsica (Schaus, 1896) & 4 & & & \\
\hline 22. Eucereon rosa (Walker, 1854) & 22 & 19 & 37 & \\
\hline 23. Eucereon sp.1 & & & 13 & \\
\hline 24. Eucereon sp.2 & 158 & 10 & & \\
\hline 25. Euceriodes pallada (Druce, 1906) & & & 11 & \\
\hline 26. Nelphe confinis (Herrich-Schäffer, 1855) & & 2 & 3 & \\
\hline 27. Philoros affinis (Rothschild, 1912) & 22 & 43 & 1 & 152 \\
\hline 28. Pseudohyaleucerea vulnerata Butler, 1875 & 3 & 3 & 14 & 1 \\
\hline 29. Sciopsyche tropica (Walker, 1854) & & & 1 & \\
\hline 30. Theages leucophaea Walker, 1855 & 1 & 4 & 1 & \\
\hline 31. Tipulodes ima Boisduval, 1832 & & & & 2 \\
\hline \multicolumn{5}{|l|}{ Euchromiini } \\
\hline 32. Aristodaema hanga (Herrich-Schäffer, [1854]) & 5 & 6 & & \\
\hline 36. Dycladia lucetius (Stoll, 1781) & 1 & 3 & & 8 \\
\hline 37. Eurota helena (Herrich-Schäffer, 1854) & & 1 & & 6 \\
\hline 38. Eurota herricki Butler, 1876 & 2 & & & 7 \\
\hline 39.Eurota nigricincta Hampson, 1907 & & & & 1 \\
\hline 40. Eurota picta (Herrich-Schäffer, 1853) & & 3 & & \\
\hline 41. Eurota schausi Hampson, 1898 & 28 & & & \\
\hline 42. Homoeocera acuminata (Walker, 1856) & 2 & 1 & & \\
\hline 43. Ichoria chalcomedusa Druce, 1893 & 1 & & 9 & \\
\hline 44. Macrocneme sp. & & & 13 & 14 \\
\hline 45. Mallodeta clavata (Walker, 1854) & & & 2 & \\
\hline 46. Mesothen desperata (Walker, 1856) & & 2 & 11 & \\
\hline 47. Mesothen sp. & & & 4 & \\
\hline 48. Mirandisca harpalyce (Schaus, 1892) & & 1 & 1 & \\
\hline 49. Napata castra Hampson, 1898 & 29 & & & \\
\hline 50. Napata sp. & 1 & & & \\
\hline 51. Neotrichura penates Druce, 1897 & & & 21 & \\
\hline 52. Paraethria triseriata (Herrich-Schäffer, 1855) & 8 & 2 & 2 & \\
\hline 53. Paramya flavia Schaus, 1898 & 52 & 2 & 2 & \\
\hline 54. Pheia sp. & & 1 & 26 & \\
\hline 55. Phoenicoprocta analis Schrottky, 1909 & & & 52 & \\
\hline 56. Phoenicoprocta teda (Walker, 1854) & & & & 1 \\
\hline 57. Psilopleura sanguipuncta Hampson, 1898 & 17 & & & 4 \\
\hline 58. Rhynchopyga meisteri (Berg, 1883) & 1 & & 1 & \\
\hline 59. Saurita cassandra (Linnaeus, 1758) & & & 1 & \\
\hline 60. Euchromiini sp. 1 & & & 2 & \\
\hline 61. Euchromiini sp.2 & & & 1 & \\
\hline 62. Euchromiini sp.3 & 2 & 2 & & \\
\hline 63. Euchromiini sp.4 & 9 & & & \\
\hline 64. Euchromiini sp.5 & & & & 2 \\
\hline \multicolumn{5}{|l|}{ Pericopini } \\
\hline 65. Calodesma collaris (Drury, 1782) & & & 6 & \\
\hline 66. Calodesma dioptis (R. Felder, 1874) & & & 2 & \\
\hline 67. Dysschema amphissa (Geyer, 1832) & & 3 & 154 & \\
\hline
\end{tabular}


Tabela I. Continuação.

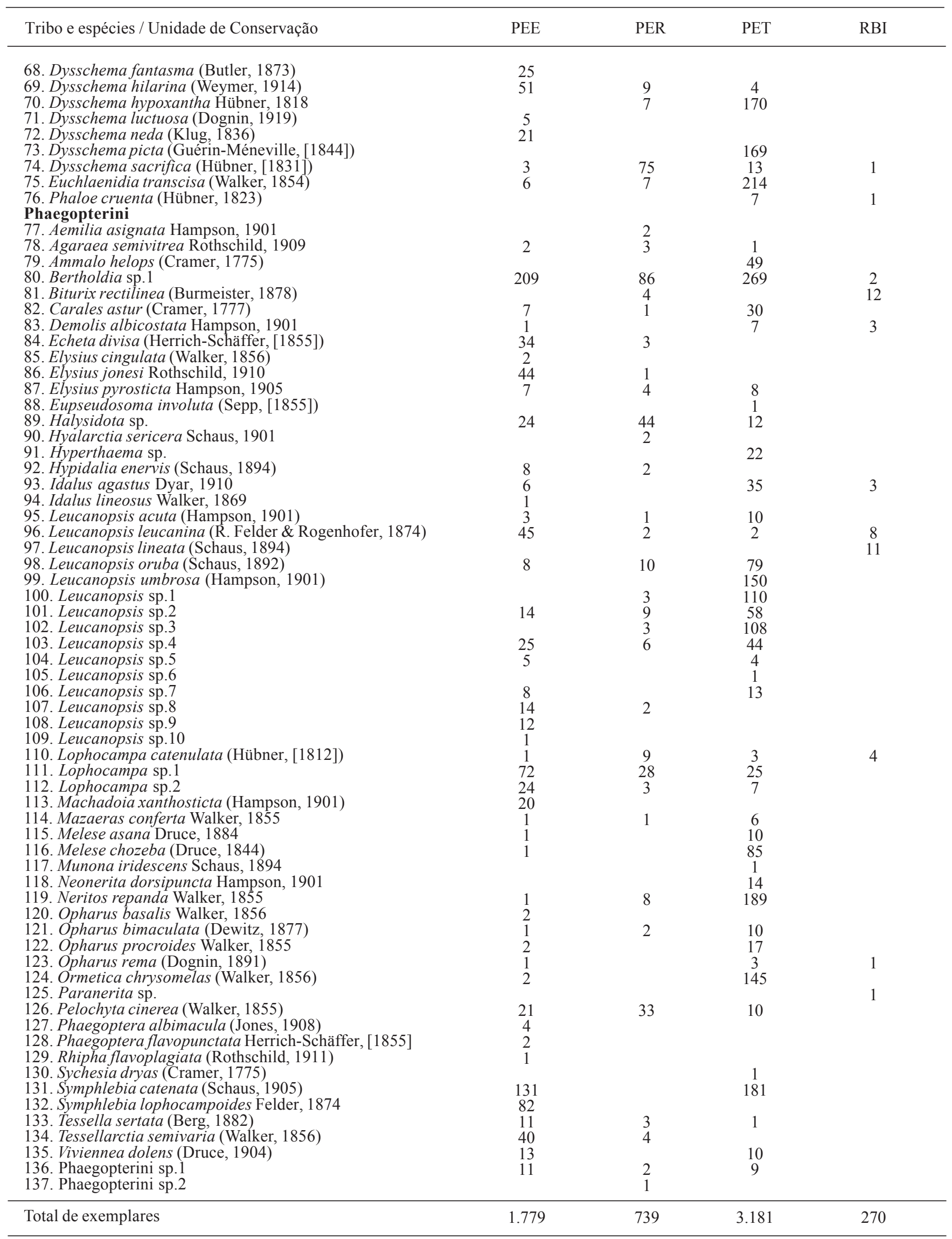


Tabela II. Número de espécies por tribos de Arctiinae capturadas com armadilha luminosa em quatro Unidades de Conservação Estaduais do Rio Grande do Sul (PEE - Parque Estadual do Espigão Alto, PER Parque Estadual de Rondinha, PET - Parque Estadual do Turvo e RBI - Reserva Biológica do Ibirapuitã). Número total de Arctiinae neste estudo (Total UC's) e registrado para o Rio Grande do Sul (Total RS).

\begin{tabular}{lcccccc}
\hline & PEE & PER & PET & RBI & Total UC's & Total RS \\
\hline Arctiini & 5 & 5 & 4 & 3 & 7 & 31 \\
Callimorphini & 0 & 0 & 0 & 1 & 1 & 1 \\
Ctenuchini & 14 & 13 & 16 & 5 & 23 & 80 \\
Euchromiini & 16 & 12 & 17 & 8 & 33 & 61 \\
Pericopini & 6 & 5 & 9 & 2 & 12 & 25 \\
Phaegopterini & 45 & 30 & 40 & 9 & 61 & 130 \\
Total & 86 & 65 & 86 & 28 & 137 & 328 \\
\hline
\end{tabular}

decorreu do insignificante relacionamento das espécies de Arctiinae com cultivos agrícolas (Costa Lima, 1950; Gallo et al. 2002; Silva et al. 1968). O reduzido número de espécies restritas a $\mathrm{RBI}(\mathrm{n}=8)$ deve estar relacionado com o ambiente campestre característico do Bioma Campos Sulinos, o que já foi evidenciado em trabalhos mais extensos (Teston \& Corseuil 2004), como conseqüência da maior diversidade vegetal, indicando que a riqueza de Arctiinae tende ser maior em áreas florestadas. Com relação às espécies de ocorrência simultânea em dois locais ( $\mathrm{n}=37$ ) (Tabela III) observouse que os maiores valores foram encontrados entre unidades da Mata Atlântica e os menores quando confrontadas áreas dos dois biomas. De forma semelhante, o número de espécies de ocorrência simultânea em três unidades $(\mathrm{n}=31)$ foi muito mais expressivo quando se analisaram os dados das três unidades pertencentes à Mata Atlântica. Tais resultados eram esperados em função das semelhanças entre as unidades que pertencem ao mesmo Bioma (Brasil 2000). Tal comportamento também foi observado com a comunidade de Noctuidae porém, de maneira menos pronunciada (Specht et al. 2005).

O percentual de arctíneos de ocorrência simultânea entre todas as unidades (7,3\%) é surpreendentemente semelhante ao valor 7,2\% encontrado para Noctuidae (Specht et al. 2005). Destes, apenas quatro (A. heber, A. terra, P. affinis e Bertholdia sp.1) foram representados por mais de 100 exemplares.

A ocorrência de 31 arctiíneos ainda não identificados ao nível específico (Tabela I) indica que o número de representantes ocorrentes no Estado é maior do que os 328 já identificados (Teston \& Corseuil 2002, 2003a, 2003b, 2004) ampliando os registros para o Rio Grande do Sul e para o Brasil. Com relação a este tipo de contribuição destaca-se a primeira citação de Dysschema luctuosa (Dognin, 1919) para o Brasil (Teston \& Corseuil 2002), realizada em função das coletas realizadas no Parque Estadual do Espigão Alto (PEE), ratificando a relevância das Unidades de Conservação como refúgio para as espécies.

Diversos estudos têm demonstrado grande correlação entre a diversidade de invertebrados e a da vegetação (Crisp et al. 1998), a de vertebrados (Blair 1999) e, principalmente, de
Tabela III. Número de espécies de Arctiinae, exclusivas e/ou de ocorrência simultânea, capturadas com armadilha luminosa em quatro Unidades de Conservação Estaduais do Rio Grande do Sul (PEE - Parque Estadual do Espigão Alto, PER - Parque Estadual de Rondinha, PET - Parque Estadual do Turvo e RBI - Reserva Biológica do Ibirapuitã).

\begin{tabular}{|c|c|c|c|c|c|}
\hline & & PEE & PER & PET & RBI \\
\hline \multirow{4}{*}{ Um local } & 23 & & & & \\
\hline & 4 & & & & \\
\hline & 24 & & & & \\
\hline & 8 & & & & \\
\hline
\end{tabular}
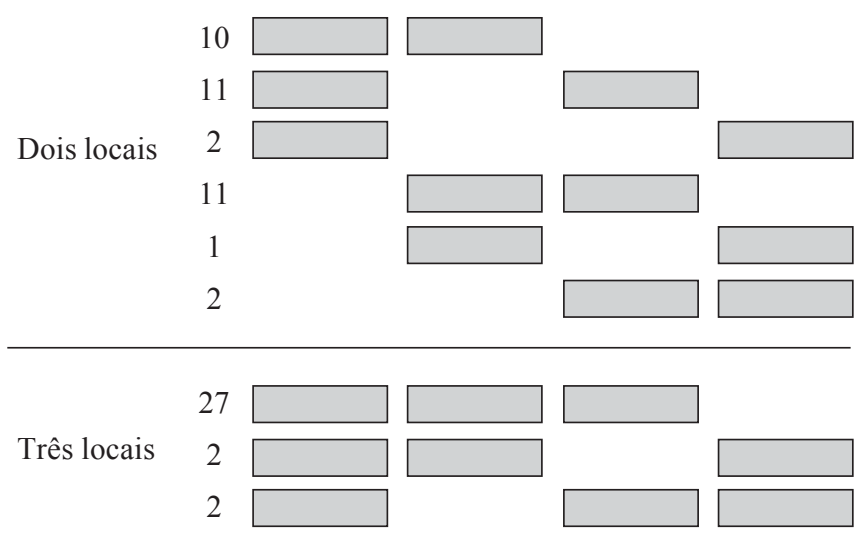

Todos $10 \square \square \square \square$

outros insetos da mesma ordem (e.g. Hamond \& Miller 1998; Intachat \& Woiwod 1999; Landau et al. 1999; Kitching et al. 2000). Entretanto, Marinoni \& Dutra (1996) e Marinoni et al. (1997) encontraram diversas diferenças entre Ctenuchini e Saturniidae coletados em oito localidades no Paraná. As semelhanças entre as comunidades de Arctiinae e Noctuidae podem ser atribuídas à proximidade taxonômica apresentando estruturas e hábitos similares por pertencerem à mesma superfamília (Kitching \& Rawlins 1999).

Agradecimentos. A Alexandre Carvalho, da Magna Engenharia LTDA, pela operacionalização e viabilização dos trabalhos de campo. A Hervé de Toulgoët, do Muséum National d'Histoire Naturelle, Paris, pelo auxílio na determinação de algumas espécies. Aos curadores, Alexandre Soares, Carlos Campaner, Edeutraud Piorezan, Eduardo J. Ely e Silva, Élvia E. S. Vianna, Fernando R. Meyer, Fernando Z. da Cruz, Luciano Moura, Olaf H.H. Mielke, Sebastião J. de Oliveira e Vera R. S. Wolff, que possibilitaram o exame das coleções entomológicas, e a suas respectivas instituições.

\section{REFERÊNCIAS}

Bittencourt, M. A. L.; L. Boaretto; I. Serafim \& E. Berti Filho. 2003.

Fauna de lepidóptera associada a um ecossistema natural no Estado de São Paulo. Arquivos do Instituto Biológico 70: 85-87.

Blair, R. B. 1999. Birds and butterflies along an urban gradient: surrogate 
taxa for assessing biodiversity? Ecological Applications 9: 164170 .

Brasil. 2000. Avaliação e ações prioritárias para a conservação da biodiversidade da Mata Atlântica e Campos Sulinos. Brasilia, Ministério do Meio Ambiente / Secretaria da Biodiversidade e Florestas. $40 \mathrm{p}$.

Butler, L.; V. Kondo; E. M. Barrows \& E. C. Townsend. 1999. Effects of weather conditions and trap types on sampling for richness and abundance of forest macrolepidoptera. Environmental Entomology 28: 795-811.

Camargo, A. J. A. de. 1999. Estudo comparativo sobre a composição e a diversidade de lepidópteros noturnos em cinco áreas da Região dos Cerrados. Revista Brasileira de Zoologia 16: 369-380.

Costa Lima, A. da. 1950. Insetos do Brasil. Lepidópteros. $2^{\text {a }}$ parte Rio de Janeiro: Imprensa Nacional, $420 \mathrm{p}$.

Crisp, P. N.; J. M. Dickinson \& G. W. Gibbs. 1998. Does native invertebrate diversity reflect native plant diversity? A case study from New Zealand and implications for conservation. Biological Conservation 83: $209-220$

Dorval, A.; J. C. Zanuncio; J. M. M. Pereira \& W. L. Gasperazzo. 1995. Análise faunística de Eupseudosoma aberrans Schaus, 1905 e Eupseudosoma involuta (Sepp, 1852) (Lepidoptera: Arctiidae) em Eucalyptus urophylla e Eucalyptus cleziana na região de Montes Claros, Minas Gerais. Revista Árvore 19: 228-240.

Draudt, M. 1915-1917. Syntomidae, p. 33-230. In: A. Seitz (ed.). Die Gross-Schmetterlinge der Erde. II. Abteilung: Die GrossSchmetterlinge des Amerikanischen Faunengebietes. 6. Band. Die Amerikanischen Spinner und Schwärmer. Stuttgart, Alfred Kernen, 497 p.

Ferreira, P. S. F.; A. S. Paula \& D. S. Martins. 1995. Análise faunística de Lepidoptera Arctiidae em área de reserva natural remanescente de floresta tropical em Viçosa, Minas Gerais. Anais da Sociedade Entomológica do Brasil 24: 123-133.

Frost, S. W. 1957. The Pennsylvania Insect Light Trap. Journal of Economic Entomology 50: 287-292.

Fry, R. \& P. Waring. 1996. A guide to moth traps and their use. The Amateur Entomologist 24: 1-60.

Gallo, D.; O. Nakano; S. Silveira Neto; R. P. L. Carvalho; G. C. de Baptista; E. Berti Filho; J. R. P. Parra; R. A. Zucchi; S. B. Alves; J. D. Vendramim; L. C. Marchini; J. R. S. Lopes \& C. Omoto. 2002 Entomologia Agrícola. Piracicaba, FEALQ, 920 p.

Hammond, P. C. \& J. C. Miller. 1998. Comparison of the biodiversity of Lepidoptera within three forested ecosystems. Annals of the Entomological Society of America 91: 323-328.

Hampson, G. F. 1898. Catalogue of the Lepidoptera Phalaenae in the British Museum. Volume 1. Catalogue of the Syntomidae in the collection of the British Museum. London, Taylor and Francis, 559 p.

Hampson, G. F. 1901. Catalogue of the Lepidoptera Phalaenae in the British Museum. Volume 3. Catalogue of the Arctiadae (Arctianae) and Agaristidae in the collection of the British Museum. London, Taylor and Francis, 690 p.

Hampson, G. F. 1914. Catalogue of the Lepidoptera Phalaenae in the British Museum. Supplement. Volume I.: Catalogue of the Amatidae and Arctiadae (Nolinae and Lithosianae) in the collection of the British Museum. London, Taylor and Francis, $858 \mathrm{p}$

Hampson, G. F. 1920. Catalogue of the Lepidoptera Phalaenae in the British Museum. Supplement. Volume II.: Catalogue of the Lithosiadae (Arctianae) and Phalaenoididae in the collection of the British Museum. London, Taylor and Francis, $619 \mathrm{p}$.

Hering, M. 1925. Pericopinae, p. 425-455. In: A. Seitz (ed.). Die Gross-Schmetterlinge der Erde. II. Abteilung: Die GrossSchmetterlinge des Amerikanischen Faunengebietes. 6. Band. Die Amerikanischen Spinner und Schwärmer. Stuttgart, Alfred Kernen, 497 p.

Hilty, J. \& A. Merenlender. 2000. Faunal indicator taxa selection for monitoring ecosystem health. Biological Conservation 92: 185197.

Intachat, J. \& I. P. Woiwod. 1999. Trap design for monitoring moth biodiversity in tropical rainforests. Bulletin of Entomological Research 89: 153-163.

Jacobson, N. L. \& S. J. Weller. 2002. A cladistic study of the Arctiidae (Lepidoptera) by using characters of immatures and adults. Lanham, Thomas Say Publications in Entomology (Monograph)/ Entomological Society of America, 98 p.

Kitching, I. J. \& J. E. Rawlins. 1999. The Noctuoidea, p. 355-401. In: N. P. Kristensen (ed. vol.). Lepidoptera, Moths and Butterflies. Volume 1: Evolution, Systematics, and Biogeography. In: M. FISCHER (ed.). Handbook of Zoology. Volume IV Arthropoda: Insecta. Berlin, de Gruyter, 491 p.

Kitching, R. L.; A. G. Orr; L. Thalib; H. Mitchell; M. S. Hopkins \& A. W. Graham. 2000. Moth assemblages as indicators of environmental quality in remnants of upland Australian rain forest. Journal of Applied Ecology 37: 284-297.

Landau, B.; D. Prowell \& C. E. Carlton. 1999. Intensive versus longterm sampling to assess lepidopteran diversity in southern mixed mesophytic forest. Annals of the Entomological Society of America 92: 435-441.

Lübeck, G. M.; J. V. Oliveira \& R. P. Almeida. 1995. Análise faunística de lepidópteros coletados em duas comunidades agrícolas na Zona da Mata norte de Pernambuco. Anais da Sociedade Entomológica do Brasil 24: 353-370.

Marinoni, R. C. \& R. R. C. Dutra. 1996. Levantamento da fauna entomológica do Estado do Paraná. II. Ctenuchidae (Lepidoptera). Revista Brasileira de Zoologia 13: 435-461.

Marinoni, R. C.; Dutra, R. R. C. \& M. M. Casagrande. 1997. Levantamento da fauna entomológica do Estado do Paraná. III Saturniidae (Lepidoptera). Revista Brasileira de Zoologia 14: 473-495.

Muirhead-Thompson, R.C. 1991. Trap responses of flying insects. The influence of trap desing on capture efficiency. London, Academic Press, 287 p.

Pereira, J. M. M.; J. C. Zanuncio; J. H. Schoereder \& G. P. Santos. 1995. Agrupamento de oito povoamentos florestais em relação à fauna de lepidópteros daninhos ao eucalipto, através de análise de agrupamento. Revista Brasileira de Entomologia 39: 647-652.

Seitz, A. 1915-1925. Die Gross-Schmetterlinge der Erde. II. Abteilung: Die Gross-Schmetterlinge des Amerikanischen Faunengebietes. 6. Band. Die Amerikanischen Spinner und Schwärmer. Stuttgart, Alfred Kernen, 497 p.

Silva A. G. D’ A.; C. R. Gonçalves; D. M. Galvão; A. J. L. Gonçalves; J. Gomes; N. M. Silva \& L. Simoni. 1968. Quarto catálogo dos insetos que vivem nas plantas do Brasil, seus parasitos e predadores. Tomo 1. Parte 2. Rio de Janeiro, Ministério da Agricultura, $622 \mathrm{p}$.

Specht, A.; J. A. Teston; R. A. Di Mare \& E. Corseuil. 2005. Noctuídeos (Lepidoptera, Noctuidae) coletados em quatro Áreas Estaduais de Conservação do Rio Grande do Sul. Revista Brasileira de Entomologia 49: 130-140.

Teston, J. A. \& E. Corseuil. 2002. Arctiinae (Lepidoptera, Arctiidae) ocorrentes no Rio Grande do Sul, Brasil. Parte I. Pericopini. Biociências 10: 79-86.

Teston, J. A. \& E. Corseuil. 2003a. Arctiinae (Lepidoptera, Arctiidae) ocorrentes no Rio Grande do Sul, Brasil. Parte II. Arctiini, Callimorphini e Phaegopterini. Biociências 11: 69-80.

Teston, J. A. \& E. Corseuil. 2003b. Arctiinae (Lepidoptera, Arctiidae) ocorrentes no Rio Grande do Sul, Brasil. Parte III. Ctenuchini e Euchromiini. Biociências 11: 81-90.

Teston, J. A. \& E. Corseuil. 2004. Diversidade de Arctiinae (Lepidoptera, Arctiidae) capturados com armadilha luminosa, em seis comunidades do Rio Grande do Sul, Brasil. Revista Brasileira de Entomologia 48: 77-90.

Watson, A. 1971. An illustrated catalog of the Neotropic Arctiinae types in the United States National Museum (Lepidoptera: 
Arctiidae) part I. Smithsonian Contributions to Zoology 50: $1-361$.

Watson, A. 1973. An illustrated catalog of the Neotropic Arctiinae types in the United States National Museum (Lepidoptera: Arctiidae) part II. Smithsonian Contributions to Zoology 128: 1-160.

Watson, A. 1975. A reclassification of the Arctiidae and Ctenuchidae formerly placed in the thyretid genus Automolis Hübner (Lepidoptera). With notes on warning coloration and sound. Bulletin of the British Museum Natural History (Entomology) 25: 1-104. [Supplement]

Watson, a. 1980. A revision of the Halysidota tessellaris species-group (Halysidota sensu stricto) (Lepidoptera: Arctiidae). Bulletin of the British Museum Natural History (Entomology) 40: 1-65.
Watson, A.; D. S. Fletcher \& I. W. B. Nye. 1995. Noctuoidea: Arctiidae, Cocytiidae, Ctenuchidae, Dilobidae, Dioptidae, Lymantriidae, Notodontidae, Strepsimanidae, Thaumetopoeidae \& Thyretidae. Volume 2. In: I. W. B. Nye (ed.). The generic names of moths of the world. Reprinted. London, The Natural History Museum, 228 p. Watson, A. \& D. T. Goodger. 1986. Catalogue of the Neotropical Tiger-moths. Occasional Papers on Systematic Entomology 1: $1-71$.

Weller, S. J.; R. B. Simmons; R. Boada \& W. E. Conner. 2000. Abdominal modifications occurring in wasp mimics of the CtenuchineEuchromiini clade (Lepidoptera: Arctiidae). Annals of the Entomological Society of America 93: 920-928. 